\title{
Global Maritime Surveillance by Airliner-Based AIS Detection: Preliminary Analysis
}

\author{
Simon Plass, Robert Poehlmann, Romain Hermenier and \\ Armin Dammann \\ German Aerospace Center (DLR), Institute of Communications and Navigation \\ Oberpfaffenhofen, 82234 Wessling, Germany \\ E-mail: simon.plass@dlr.de
}

\begin{abstract}
Demands on security, safety, and environmental protection in worldwide shipping are steadily increasing. Shipboard broadcast transponders based on the Automatic Identification System (AIS) can be easily detected close to coast or waterway areas. Satellite-based AIS receivers detect globally but are limited in high-density traffic areas. This paper investigates the challenges and performance of AIS detection on aircraft at altitudes between $8500 \mathrm{~m}$ and $10000 \mathrm{~m}$. During flight trials over sea and land AIS signals were recorded. Post-processing of the recorded data allows the evaluation but also faces the challenges due to the nature of overlapping AIS signals at the aircraft. A comparison of detected signals at the aircraft with received AIS signals on the ground is given including the evaluation of the reception footprint of the aircraft. Finally, a concept for worldwide AIS detection via airliners is presented. The study shows the potential for global complementary surveillance coverage via airliner-based AIS detection.
\end{abstract}

Notes:

Accepted for publication in The Journal of Navigation published by Cambridge University Press. This article will be published in a revised form and copyright holder is Cambridge University Press

See also: journals.cambridge.org/jid_NAV

1. INTRODUCTION. The purpose of the Automatic Identification System (AIS) is the improvement of maritime safety and the efficiency of navigation, safety of life at sea and the protection of the marine environment (IALA, 2003). In contrast to passive systems like radar, each ship needs to transmit information about itself continuously. Vessels automatically exchange their Global Navigation Satellite System (GNSS) position, heading, speed, and unique ship identification number. The data is gathered at a receiver and can be used to feed a graphical display showing nearby ships. The device can be accessed by the ship's captain as an aid for safe navigation.

The system was standardised and developed in the 1990s and became mandatory for all new ships, passenger ships, tankers over 300 Gross Tonnes (GT) engaged in international traffic in 2004. In 2008, it was extended to cargo ships of 500 GT or more on international voyages (IMO, 2002). There are two different types of transceivers available: Class A for mandatory equipment and Class B for smaller ships that can apply AIS by choice. The AIS system operates on two reserved channels in the maritime Very High Frequency (VHF) band. Currently, about 60000 ships are equipped with AIS Class A devices globally. Reception of VHF signals requires line-of-sight. Due to the curvature of the earth, the line-of-sight for ship-to-ship communications is typically $40-60 \mathrm{~km}$, depending on the antenna height. The same limit applies to receivers on land, used by e.g., port authorities for Vessel Traffic Services (VTS). There is a high demand for global coverage to enable surveillance, environmental protection, anti-piracy, and other applications. 
In order to overcome the limitation to coastal areas, work has been done to develop AIS receivers for satellites (Wahl and Høye, 2003), (Wahl et al., 2005), (Carson-Jackson, 2012). Although omnidirectional antennas are being used on board, signal power is high enough to allow AIS signal reception for orbits up to $1000 \mathrm{~km}$ altitude (Eriksen et al., 2006). However, space-based AIS faces other problems compared with terrestrial AIS receivers.

The AIS signal design is based on Self-organised Time-Division MultipleAccess (SOTDMA) representing a self-organised system between neighbouring AIS-shipsystems regarding the usage of time slots for information transmission. Therefore, individual AIS cells are formed seen from each ship. At an altitude of $650 \mathrm{~km}$ the field of view of a satellite is roughly $5000 \mathrm{~km}$ in diameter. Therefore the satellite's footprint covers numerous SOTDMA organisation areas. Ships in different cells cannot see each other, and thus, they do not organise time slot assignments among each other. This results in packet collisions, especially in areas with high ship density. Furthermore with only a few satellites the shipsatellite contact is limited to once or twice a day (Cervera et al., 2011). It has been discovered that space-based AIS is feasible for areas with low ship density. However, regarding highly populated European waters with about 6200 AIS equipped vessels, the ship detection probability could drop towards zero. One way to address this problem is adapting the AIS specification to prepare AIS systems for reception from space (Høye et al., 2008). This resulted in an easy-to-implement Long-Range AIS (LR-AIS) with its own two VHF channels defined at the International Telecommunication Union (ITU) World Radio Conference 2012. The total number of bits per message has been compressed to allow increased propagation delays and the transmission interval is set to $180 \mathrm{~s}$ (ITU-R M.1371-4, 2010). The probability of overlapping LR-AIS is significantly reduced when seen at the satellite. Nevertheless, even with a large footprint of $5000 \mathrm{~km}$ diameter the chance of detecting a LR-AIS signal of one ship over a possible 4-5 transmissions per orbital period is very low. This does not take into account any self-shadowing by the ship itself or limited transmission power when ship and satellite are arranged one above the other due to the already deployed antennas. Future AIS satellites with smaller footprints to reduce the viewing of interfering SOTDMA cells will also suffer from these low transmission intervals. As an example, the recently (June 2014) launched AIS satellite AISat-1 of the German Aerospace Center is equipped with a $4.2 \mathrm{~m}$ long high-gain helix antenna providing a focused footprint of about $700 \mathrm{~km}$ (Clazzer et al., 2014a). Furthermore, no new advanced random access schemes tailored to AIS satellite reception have yet been introduced (Clazzer et al., 2014b). Therefore the future VHF Data Exchange (VDE) link could introduce more advanced signal designs for ship monitoring (CEPT, 2013). Remaining with the current system, interference mitigation could be used to improve the detection rates (Prevost et al., 2012; Burzigotti et al., 2010) and also algorithms to infer the possible route from incomplete satellite AIS data (Changqing, 2013) are envisaged.

Another approach for global monitoring of ships via the AIS system could be the usage of airliners already in operation (Plass and Hermenier, 2014). These aircraft move all over the globe in known patterns and regularity. Due to the lower flight altitude than satellites, the overall footprint is reduced by orders of magnitude increasing the detection probability. Furthermore, the general footprint of airliners could be several hundred kilometres in diameter, consequently significantly smaller than a satellite footprint. Therefore AIS detection by airliners could have a higher chance of success for AIS signal reception and easier signal processing of less overlaid AIS signals. Thus receiving AIS signals on an aircraft could close the gap between coast-based AIS signal tracking and the successful satellite AIS tracking in more remote areas achieving an overall global ship monitoring. Since aircraft are already deployed with VHF antennas, they could also be used for AIS signal detection reducing additional high costs of system implementation on the aircraft. Therefore 
AIS equipped airliners could be an opportunity to complement satellite AIS. A system combining both could feature global coverage together with reliable detection in shipping hot spots and offer high timeliness of data.

Single usage of aircraft for AIS detections in coastal areas for Search And Rescue (SAR) purposes or national coast guards is known (Ou and Zhu, 2008). Work concerning the comparison of satellite received AIS signals with airborne received AIS signals have also been published (EU PASTA MARE Project, 2010b).

In this paper a flight trial campaign is the basis for airborne received AIS signals. These are collected via a data grabber. Then, they are analysed and also compared with groundbased terrestrial received AIS information. The goal is the evaluation of detection probability and coverage analyses by AIS aircraft reception at cruising altitude. This paper merges the results of Plass et al. (2014) and Plass and Hermenier (2014) and extends them to:

- A comparison with the new and comprehensive terrestrial AIS ground network data of the German Federal Waterways and Shipping Administration;

- A new evaluation of the global ship detection probability using the measured aircraft footprint.

The paper is organised as follows. First, the measurement campaign via flight trials is illustrated. Then, the receiver design is described. Finally, AIS signals collected during the flight trial at an altitude between 8500 and $10000 \mathrm{~m}$ are analysed and compared with a terrestrial AIS ground network. Finally, a global analysis of the possible ship detection probability via airliners for AIS is given.

2. AIRCRAFT MEASUREMENT CAMPAIGN. The data to be analysed has been gathered by the German Aerospace Center (DLR) during an aircraft measurement campaign in summer 2013. The signal was received via an aircraft's experimental VHF antenna below the fuselage and then fed to an Ettus Research USRP N210 data grabber connected to a notebook computer. The aircraft's current Global Positioning System (GPS) position was recorded in parallel. Both AIS channels were recorded at once with a sampling frequency of $200 \mathrm{kHz}$ and centre frequency in between (162 MHz).

Figure 1 shows the flight path above Germany. The aircraft started in Southern Germany, passed near Hamburg, headed to the North Sea with turnaround above Helgoland, then went back over Germany and finally landed near Braunschweig/Brunswick. The altitude was between flight level (FL) 280 and FL 330 (8 500 m - 10000 m). About 46 minutes of data were recorded before the approach for landing and are the focus of the analyses within this paper. Areas with low ship density in the interior of Germany were covered as well as highdensity areas such as the ports of Hamburg and Bremerhaven, and the coastal regions of the North Sea.

3. SYSTEM AND RECEIVER DESCRIPTION. AIS uses two VHF channels which are at $161.975 \mathrm{MHz}$ and $162.025 \mathrm{MHz}$ with a channel spacing of $25 \mathrm{kHz}$. AIS devices receive both channels at a time and transmit on both channels alternately. To be independent of a base station that coordinates access to the channel, a SOTDMA scheme is used (ITU-R M.1371-4, 2010). The users within range organise themselves to use different time slots to avoid collisions. Every station builds up a slot map to keep track of slots that are used by other stations. Based on this scheme they select a free slot for their own transmissions. Each ship reports its position periodically for different vessels speeds. Gaussian Minimum-Shift Keying (GMSK) is used as modulation together with non-return-to-zero inverted (NRZI) encoding. 


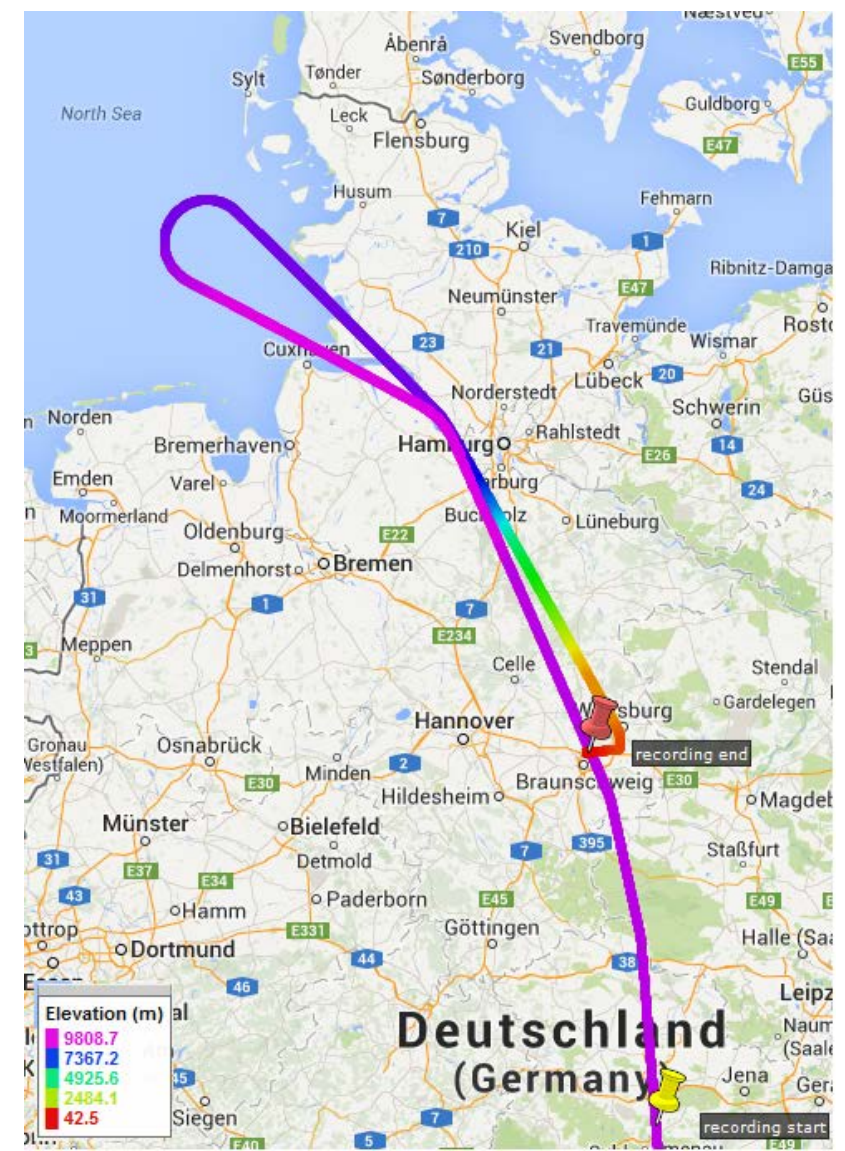

Figure 1. Flight route with indicated different altitudes.
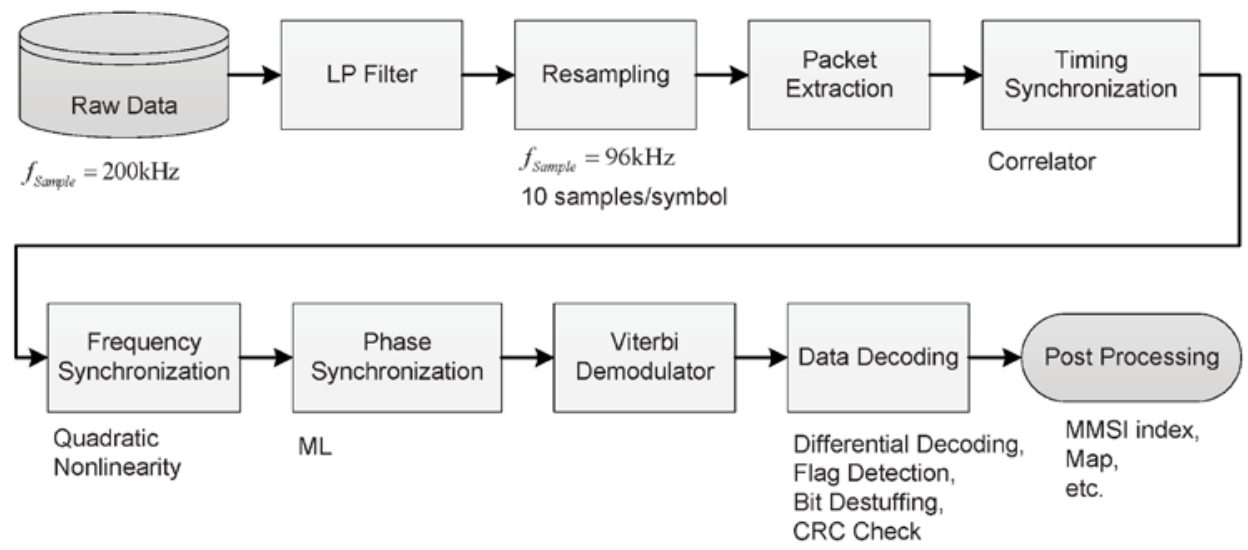

Figure 2. Architecture of implemented software receiver.

As shown in Figure 2, the receiver processes the raw data and extracts the AIS information. Both AIS channels were recorded simultaneously with a sampling frequency of $200 \mathrm{kHz}$. To begin with the channels are separated and the signal is then fed to a low-pass filter with cut-off frequency $10.6 \mathrm{kHz}$. The next step is resampling the signal in order to get an integer oversampling factor of ten, which results in a new sampling frequency of $96 \mathrm{kHz}$. The signal is then separated into packets exceeding a Signal-to-Noise Ratio (SNR) threshold of $0 \mathrm{~dB}$. For each packet delay, carrier frequency offset, and phase offset are estimated and compensated. Therefore, the receiver features combined estimation of delay and frequency offset for timing synchronisation. Frequency synchronisation is improved by a second non data-aided estimation based on a Quadratic Non-Linearity (QNL) estimator (Morelli and 
Mengali, 1998). The phase offset is also compensated using a non-data-aided approach described in Mengali and D'Andrea (1997). Next coherent Viterbi demodulation is performed (Anderson et al., 1986). After differential decoding, the payload is extracted with the help of a High-level Data Link Control (HDLC) protocol (ISO, 1993). After performing bit destuffing, the payload is separated in a data and Cyclic Redundancy Check (CRC). Finally, if the checksum matches, the message data containing ship Identity (ID), position, etc. is stored into a database. More details of the implemented receiver can be found in Poehlmann (2014) and Plass et al. (2014).

In order to evaluate the true signal quality, a SNR estimator based on the MaximumLikelihood (ML) estimation theory is implemented. In this way the Signal-to-Interferenceplus-Noise Ratio (SINR) can be estimated. A data-aided approach is taken: The received preamble of each packet is compared to an undistorted reference preamble. In Pauluzzi and Beaulieu (2000) this approach was found to fit for Additive White Gaussian Noise (AWGN) channels. Due to the existing line-of-sight between ship and aircraft this ML estimation method fits to aircraft AIS receivers under the assumption of perfect synchronisation.

For a typical aircraft speed of $840 \mathrm{~km} / \mathrm{h}$ and a maximum ship speed of 48 knots the maximum Doppler (plane and ship moving in the opposite direction) is $139 \mathrm{~Hz}$. It was verified that the frequency stability of the used receiver hardware is better than $30 \mathrm{~Hz}$. Nevertheless, the data analyses showed a larger range of the received Doppler $[-700 \mathrm{~Hz}$, $+700 \mathrm{~Hz}$ ] at the aircraft. This confirms the results in Bouny et al. (2012) and the existing specification (ITU-R M.1371-4, 2010) requires the frequency stability of the transmitter to be better than $500 \mathrm{~Hz}$. This range is apparently being covered by AIS transceivers on the market.

AIS transmitters available on the market do not only have a certain carrier frequency offset, which is compensated here, but they also generate phase noise. Another drawback is that transmitters often have a modulation index which differs from the given value 0.5 (Bouny et al., 2012). The performance of the Viterbi demodulator is affected by that. For the original usage scenario of ship-to-ship communication the link margins are so high that simple non-coherent demodulation can be applied and these effects are negligible. However, this makes the design of an optimum AIS receiver for satellite or aircraft, which involves low SNR, quite challenging. One way to deal with that would be to estimate the modulation index and adapt the receiver accordingly. That part is not taken into account in the current receiver architecture.

4. ANALYSIS OF RECORDED DATA. The whole length of the analysed data records is about 46 minutes. Figure 3 shows the flight path in purple together with ship positions from all AIS signals that have been received during that time. The red diamond markers represent the moving ships' final positions, the blue squares moored ships. Position reports received earlier are indicated with smaller red or blue markers. The large reception radius is evident. The aircraft flying over Germany could see ships near the Dutch coast as well as ships around Copenhagen. Several inland waterways are covered as well as parts of North Sea and Baltic Sea. Areas with dense shipping traffic such as Hamburg and the river Elbe, Bremen, Bremerhaven, and Kiel are included, too.

4.1. Packet Statistics and Message Types. The packet statistics in Table 1 reveal a high number of detected packets. The percentage of error-free packets is only $29 \%$. This is a first indication that a lot of packet collisions have happened. With a total of almost 179000 received packets, a good overview of the typical AIS communication traffic can be obtained. As indicated in Plass et al. (2014), with about $78 \%$ by far most of the packets are normal scheduled position reports. The second biggest percentage of $16 \%$ is position reports that were sent in response to interrogations from other vessels. 


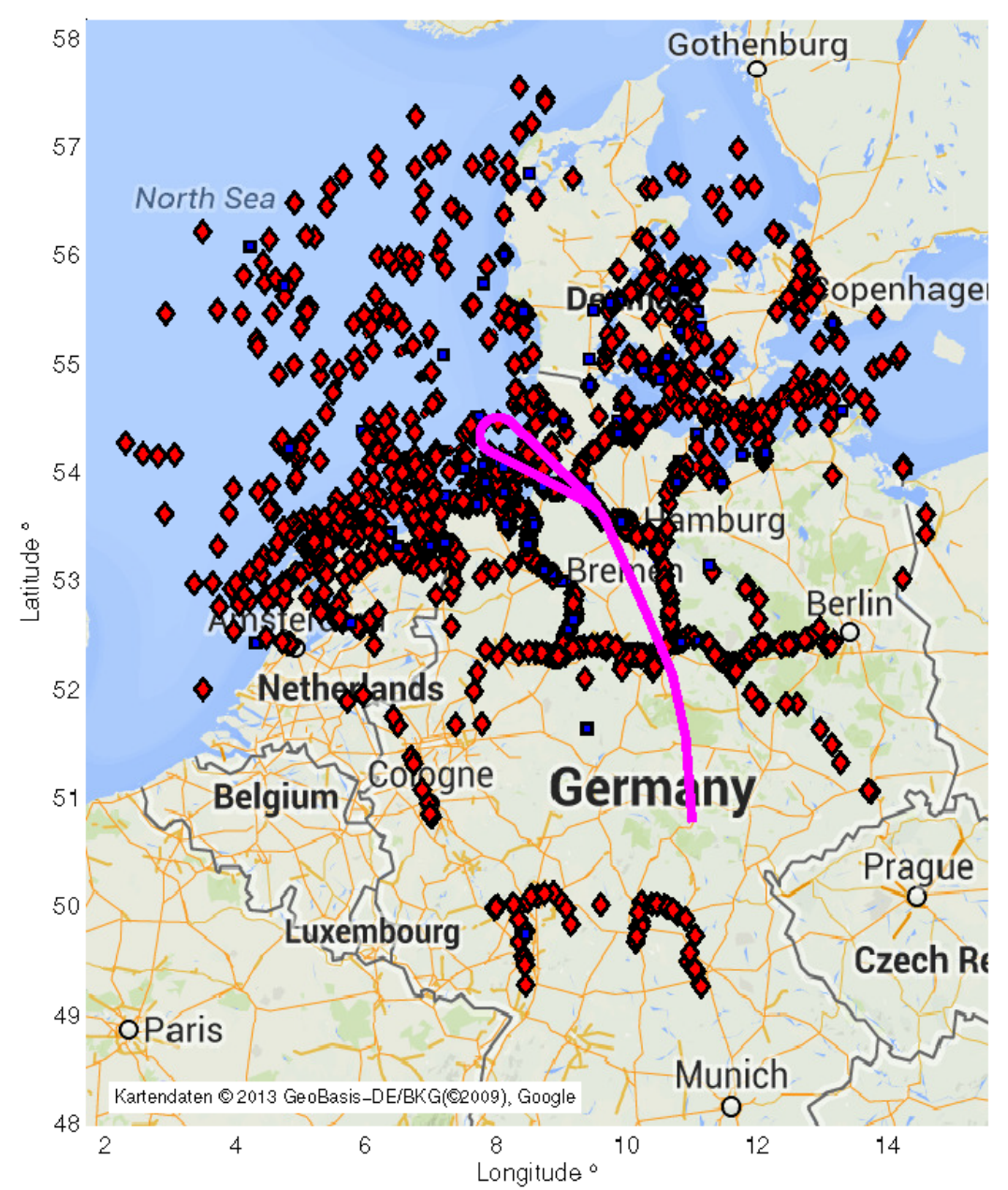

Figure 2. All detected ships on flight route within 46 minute data records.

Table 1. Statistics of 46 min recorded data during measurement campaign.

\begin{tabular}{lll}
\hline Packets detected & 178788 & $100 \%$ \\
Packets with correct CRC & 51918 & $29.0 \%$ \\
Packet detection rate & & $3823 \min ^{-1}$ \\
Valid packet detection rate & & $1110 \mathrm{~min}^{-1}$ \\
\hline
\end{tabular}

4.2. Coverage Area. When obstructions and atmospheric effects are neglected, the aircraft coverage area is determined by assuming line-of-sight propagation conditions. The theoretical coverage area by an assumed aircraft altitude of $10 \mathrm{~km}$ and ship antenna height of $10 \mathrm{~m}$ has a radius of $368 \mathrm{~km}$ (Plass et al., 2014). 


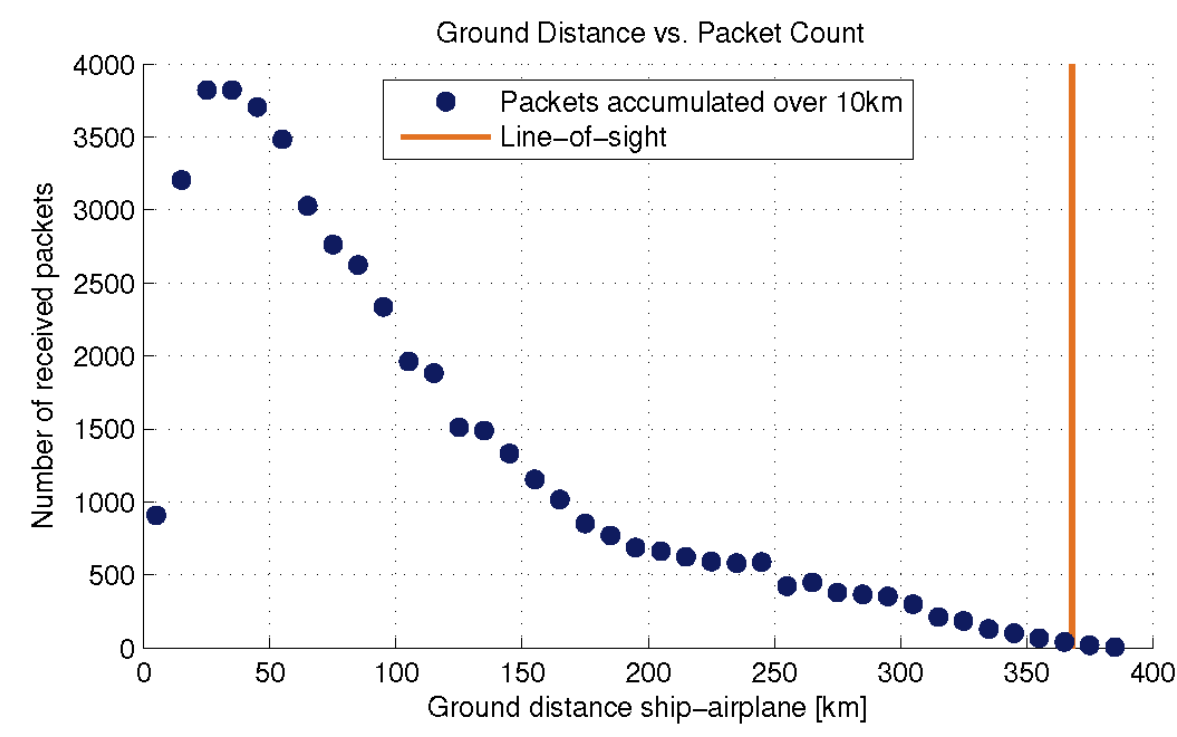

Figure 4. Number of AIS packets received with correct CRC from a certain distance.

Since most of the packets received were position reports and the GPS position of the aircraft was tracked, the hypothetical coverage area can be compared to the actual one. For that, the ground distance between ships and the aircraft, projected on ground, has been calculated. We can see from Figure 4 that the theoretical radius is actually reached, but only few packets were received at that distance. In fact, the packet count drops drastically with increasing distance. Reasons for that are packet collisions in combination with path loss. When a packet from nearby collides with one from far away, which is maybe $10-20 \mathrm{~dB}$ weaker, the first packet survives. It is worth noting that the number of detected packets drops also for low distances. This is caused by the antenna pattern, which tends to zero for the corresponding directions. Also for satellite AIS, the centre of the footprint has a low detection probability.

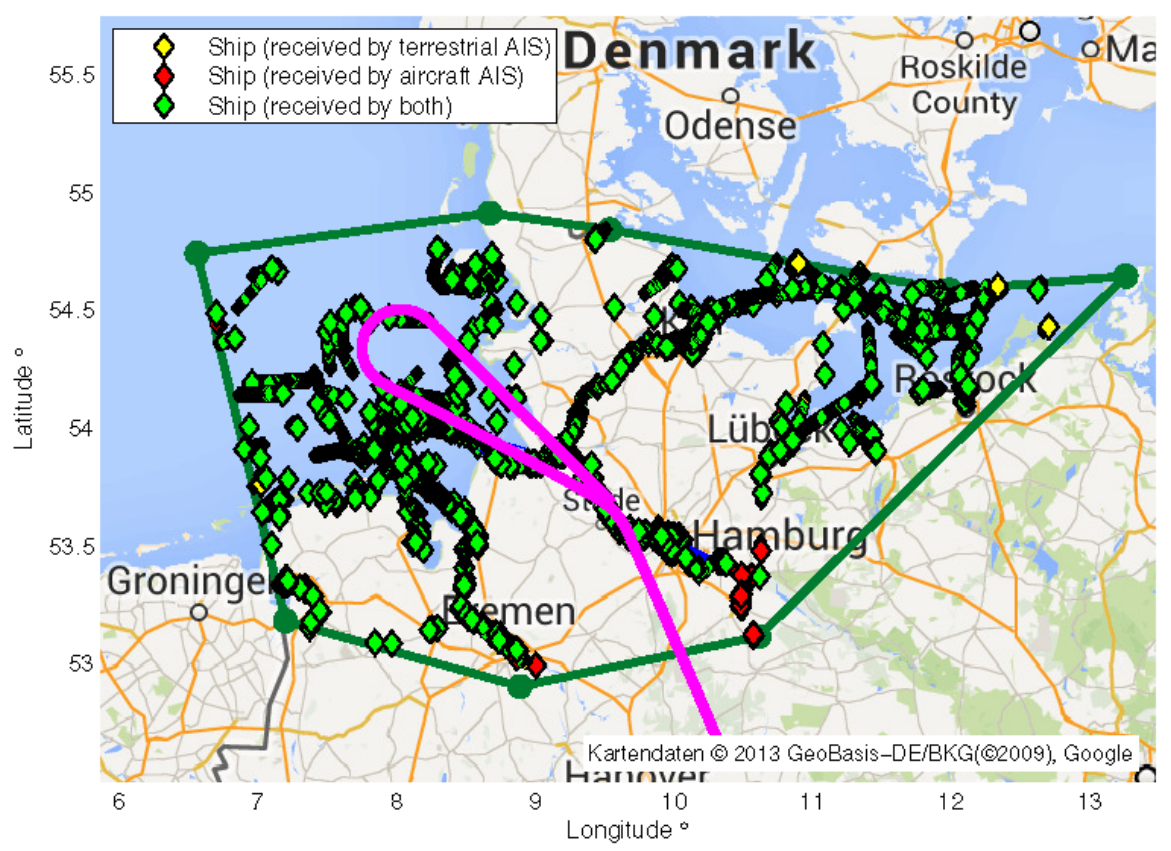

Figure 5. Comparison of terrestrial and aircraft detected AIS data. 
4.3. Comparison with Terrestrial AIS Data. Within the work of Plass et al. (2014) a limited preliminary terrestrial AIS test bed was used for comparison. This was deployed in the surroundings of Bremerhaven on the North Sea. We now show an evaluation of the detected aircraft AIS data with a full set of terrestrial AIS data provided by the German Federal Waterways and Shipping Administration over the whole country's territory. The data set used does not include inland waterways.

For comparison with the aircraft AIS, a time window of 32 minutes has been chosen in which the whole area is within the aircraft's reception radius. Figure 5 shows the area covered by the ground-based AIS receivers. The polygon was constructed taking into account the outer ships to define the reception radius of the terrestrial AIS. Within Figure 5 three types of detected ships are specified: Ships detected only by terrestrial AIS in yellow; ships detected only by aircraft AIS in red; and ships detected by both systems in green.

Looking at Table 2, we see that the terrestrial AIS has received about ten times more packets and detected only 20 more vessels out of about 1000 . All values are with regard to the defined area. The map in Figure 5 reveals that most ships were detected by both systems. There are, however, ships that were detected by only one of the systems. 41 ships were detected by aircraft exclusively due to the missing data of the inland waterways of the terrestrial data set. The aircraft on the other hand could also not detect all ships. 21 ships were only detected by the terrestrial AIS system in the outer coverage regions of aircraft's footprint in the Eastern part of German's Baltic Sea. Consequently, the closer the ship is to the aircraft the higher the detection probability. The detection probability depends on the distance between ship and aircraft, see also Figure 4. Assuming, the terrestrial AIS as reference the aircraft AIS would provide a packet detection probability of $15 \%$. Even with the used simple receiver design without any multi-user interference cancellation an averaged updated interval of $61 \mathrm{~s}$ can be achieved which gives a feasible tracking and surveillance rate.

Table 2. Comparison of aircraft and terrestrial AIS.

\begin{tabular}{l|l|l}
\hline & Aircraft AIS & Terrestrial AIS \\
\hline Valid packets detected & 33970 & 372005 \\
Packet detection rate & $1045 \mathrm{~min}^{-1}$ & $11446 \mathrm{~min}^{-1}$ \\
Vessels detected & 1070 & 1090 \\
Avg. update interval & $61 \mathrm{~s}$ & $5.5 \mathrm{~s}$ \\
\hline
\end{tabular}

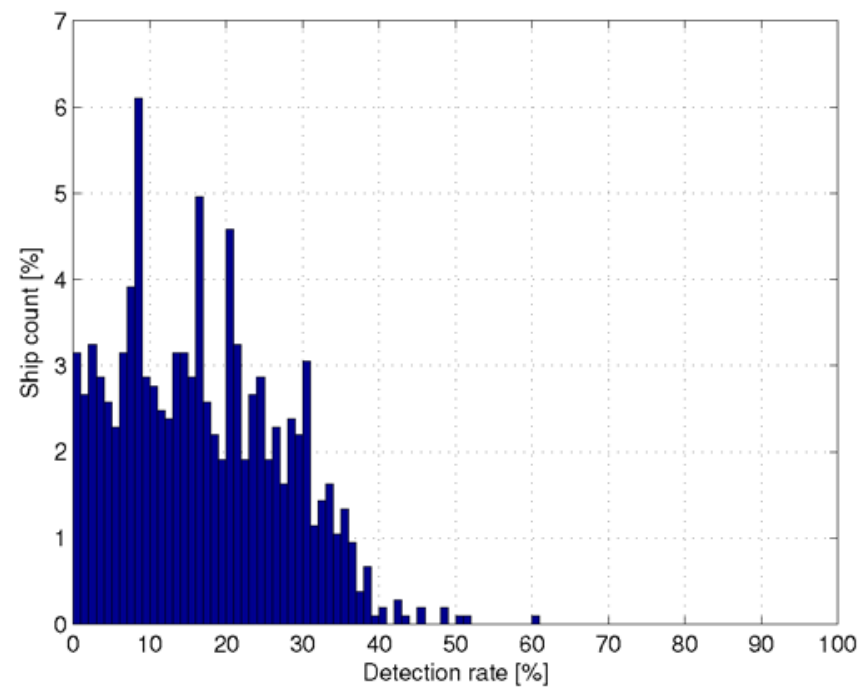

Figure 6. Distribution of the detection rate versus ship count. 
Figure 6 shows the distribution of detections rates versus the percentage of all ships. There are some individual ships with a detection rate of more than $35 \%$ but not more than $60 \%$. Nevertheless, there is no significant distribution or decrease or gain over the different detection rates.

5. AIS AIRLINER INFRASTRUCTURE. In Plass and Hermenier (2014), the principle idea of using regular commercial aircraft in operations for a global monitoring of ships was introduced. Within this section a closer look is taken at the identified aircraft footprint of the previous section. A pessimistic assumption was made in Plass and Hermenier (2014) with regards to the area covered area by aircraft's footprint being around $200 \mathrm{~km}$ in diameter in an open sea scenario. This size of footprint could hold in mainland scenarios influenced by the terrain impact as shown in Plass et al. (2013). Also the flight measurement campaign confirms the assumption of a footprint with radius between $170-180 \mathrm{~km}$ on the mainland due to the underlying terrain grade. Focusing on the starting point of the flight measurement campaign, Figure 2 shows detection of ships on inland waterways in the area of Nuremberg (north of Munich), approximately $170-180 \mathrm{~km}$ away from the aircraft's position. Nevertheless, ships are not detected in the area of Regensburg (out of range, more than $180 \mathrm{~km}$ ) on the already navigable Danube (east of Nuremberg) in contrast to ships detected already on the North Sea close to Cuxhaven more than $300 \mathrm{~km}$ away.

The newly validated footprint radius of about $370 \mathrm{~km}$ directly raises the questions: How large is the overlap of both traffic systems now? And how often can a ship see an aircraft flying over it averaged over one day?

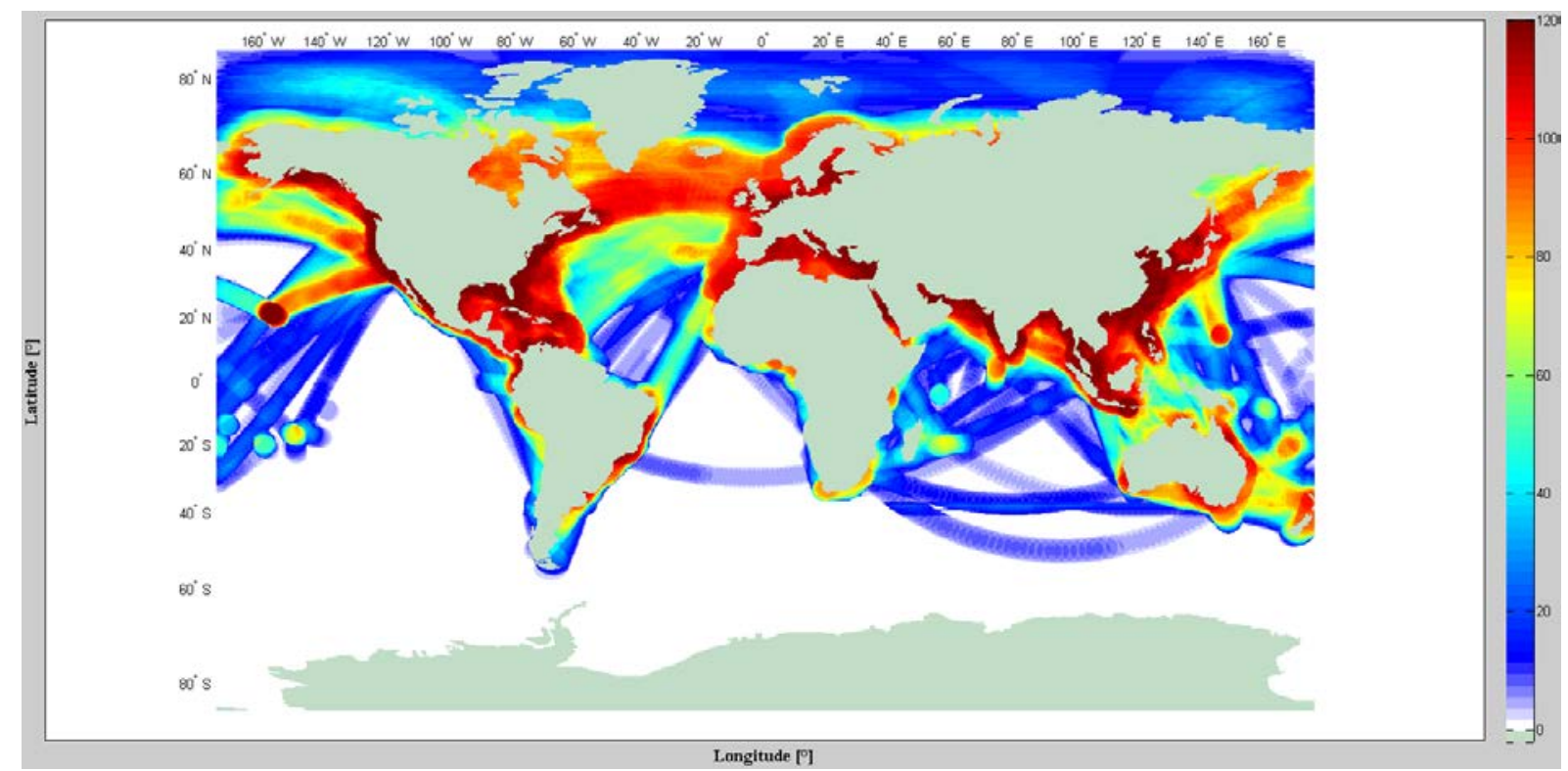

Figure 7. Airliners coverage on oceans of all commercial flights during 24 hours on 21 May 21, 2007 with an aircraft footprint of about $370 \mathrm{~km}$ radius. Colour represents the number of contacts per day with potential ships in these areas.

Figure 7 shows the simulated global coverage density at sea. Inland waterways, the Great Lakes, Caspian Sea, and the Black Sea, etc. are not considered here. The coverage intensity of each evaluated square area $\left(1^{\circ}\right.$ by $\left.1^{\circ}\right)$ over 24 hours is represented as the number of contacts within this time period. Therefore, 100\% represent 120 contacts over the day due to a 12-min snapshot frequency. Yellowish and reddish colour represents more than 70 contacts per day with potential ships in these areas. Even light bluish coloured areas have more than 
10 contacts per day. For comparison, a global coverage figure for the pessimistic footprint of $200 \mathrm{~km}$ diameter is given in the second figure of Plass and Hermenier (2014).

The problem for an evaluation of global coverage rates between ship and airline traffic is the non-existing global real time ship patterns. Global coverage could be given by satellite AIS. However, verification of successful AIS detection via satellite is not so easy to do (Brusch et al., 2011; Zhi, 2014). In Greidanus et al. (2013), a detection probability including satellite AIS and Long-Range Identification and Tracking (LRIT) were only 50\% compared by radar survey. Within these evaluations, we take advantage of freely available global data by several global satellite AIS detection sets of ship patterns by the EU PASTA MARE Project (2010a). Also in these data, the satellite AIS system used could detect about $1 / 3$ of the AIS equipped ships. Therefore, the ship patterns were weighted by a factor reaching the total worldwide number of 60000 assumed ships.

Geographically, the airliners globally cover $84 \%$ of areas with ship appearance (only 62.5\% with the pessimistic footprint (Plass and Hermenier, 2014)). In contrast to the geographical distribution, the overall AIS detection probability by airliners is now an astonishing 96.8\% (only 85.9\% with the pessimistic footprint (Plass and Hermenier, 2014)). That revisits by airliners of possible AIS detection is very high (at least once a day for this evaluation). Even $78.7 \%$ of the overflown areas have actually more than four contacts per day. These figures are summarised in Table 3. Also Figure 8 shows the difference of geographical detection coverage for the pessimistic and measured footprint. The yellow areas identify the additional coverage areas by having the measured $370 \mathrm{~km}$ radius footprint compared to the pessimistic $200 \mathrm{~km}$ radius. The brown areas denote areas not covered by airliners.

Table 3. Airliner potential global coverage for AIS detection.

\begin{tabular}{l|c|c} 
& $\begin{array}{l}\text { Pessimistic footprint of 200 km } \\
\text { diameter } \\
\text { (Plass and Hermenier, 2014) }\end{array}$ & $\begin{array}{l}\text { Measured footprint } \\
\text { of 370 km diameter }\end{array}$ \\
\hline $\begin{array}{l}\text { Geographically covered area } \\
\text { with ship appearance }\end{array}$ & $62.5 \%$ & $84.0 \%$ \\
$\begin{array}{l}\text { Percentage of all ships within } \\
\text { covered area }\end{array}$ & $85.9 \%$ & $76.8 \%$ \\
$\begin{array}{l}\text { Ships with more than 4 contacts } \\
\text { during 24 hours }\end{array}$ & $64.0 \%$ & $78.7 \%$ \\
\hline
\end{tabular}

However satellite and airliner AIS are complementary as the regions not covered by the airliners are perfectly covered by satellite AIS.

6. DISCUSSION AIS data are beneficial or even required in the following areas: vessel tracking / monitoring; traffic pattern monitoring; notice of arrival / pre-arrival notification; ship reporting; port state control; environmental protection; search and rescue; anti-piracy applications. Requirement for sufficient usage by major applications is a location update of at least every three hours (Cervera et al., 2011).

Studies show the need for 5-10 Low Earth Orbit (LEO) satellites at an altitude of $600 \mathrm{~km}$ to guarantee a target probability of ship detection of about $80-90 \%$ (Cervera et al., 2011; te Hennepe et al., 2010). Recently, the largest satellite AIS provider launched its fifth AIS satellite (exactEarth, 2013). The proposed concept of AIS detection airliner infrastructure could be used as a complementary system. Due to large airline alliances such as OneWorld or Star Alliance, global coverage with a high number of vessel contacts per day could be 
guaranteed - even a large airline (e.g., United Airlines, Lufthansa, or Air France) could cover large global areas with their own network with several ship contacts per day. Furthermore, as shown in the evaluation of the flight trials reported in this paper, the applied simple receiver design used here successfully detected all ships within its coverage area including a reasonable detection rate of 60 seconds on average. Beneficially, less overlapped AIS signals are within the smaller footprint of an aircraft. Furthermore, assuming the measured footprint of about $370 \mathrm{~km}$, even the less frequent aircraft routes around the West African Coast and from the Cape of Good Hope to Asia are adequately covered, see Figure 8.

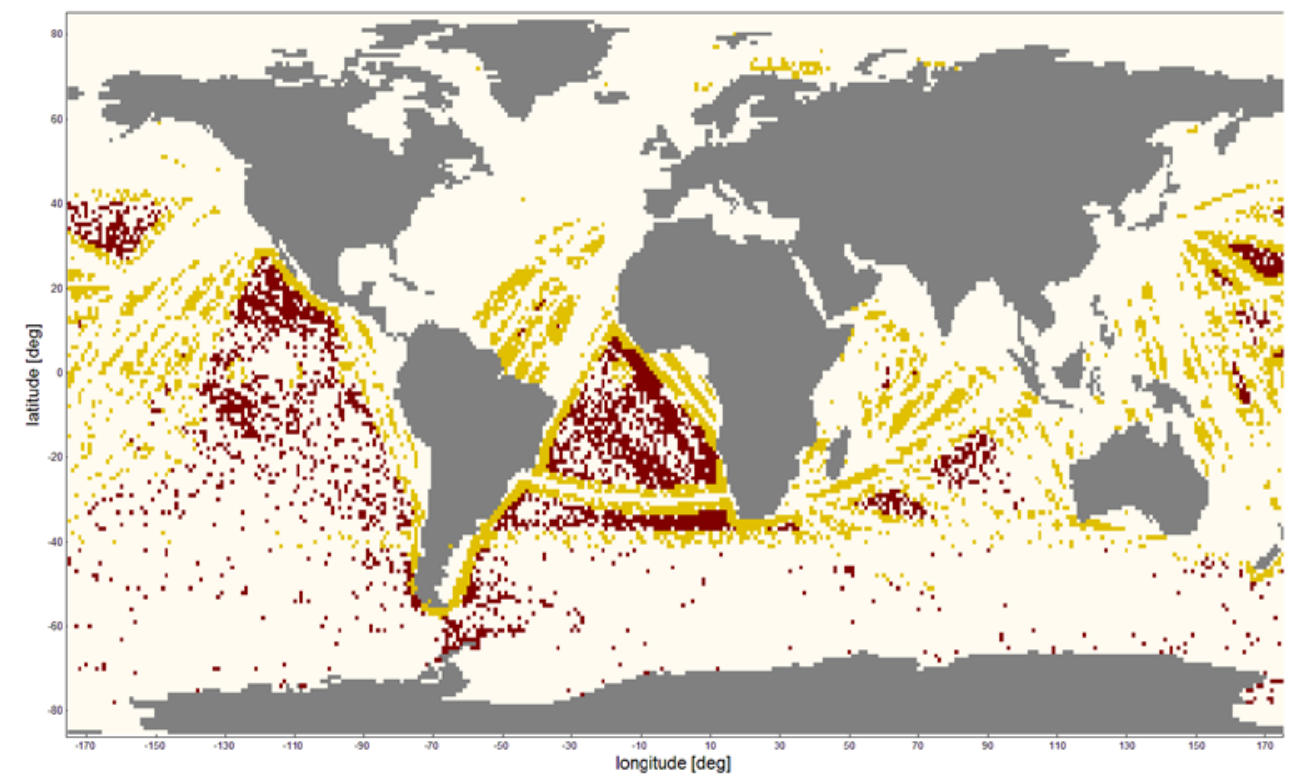

Figure 8. Additional airliners' coverage by having a footprint of about $370 \mathrm{~km}$ radius compared to $200 \mathrm{~km}$ coloured in yellow and non-covered shipping areas.

Finally, for a larger coverage and higher detection probability several integrative systems with different data sources are proposed or already exist. For example, overall situation awareness is increased by using detection data of AIS, Inmarsat and the Chinese BeiDou navigation satellite system (Yang, 2012). Other multi-source approaches can be found in Greidanus et al. (2013) and Zhi (2014). Additionally, all collected data could be fed into an Internet AIS (Filjar et al., 2005), providing global data to all users.

7. CONCLUSIONS. Within this paper a flight measurement campaign collecting AIS signals at higher altitudes was analysed. The fundamental idea is the usage of airliners gathering AIS data on a global scale. Although the described receiver architecture does not carry out interference mitigation, all ships were detected compared to terrestrial based AIS reception within the range of aircraft's signal reception.

It has been shown that a line-of-sight model is a good approximation of the actual coverage area of an aircraft in terms of AIS reception which results in a coverage footprint of about $370 \mathrm{~km}$ radius. However, the detection probability depends on the distance between ship and aircraft. Terrestrial AIS features a higher position update rate, but is limited to coastal areas. The update rate of 60 seconds on average for aircraft AIS is still high enough for surveillance and allows tracking with reasonable accuracy. 
In total, this work showed more than a 96\% AIS detection probability by airliners and about $78 \%$ of the overflown areas have more than four contacts per day on a global scale. This is an increase of more than 10\% compared to the theoretical pessimistic $200 \mathrm{~km}$ radius footprint assumption. AIS detection via airliners can close the gap of global ship monitoring between coastal and satellite systems in an easy to implement way on aircraft.

\section{ACKNOWLEDGEMENTS}

We would like to thank German Federal Waterways and Shipping Administration providing the terrestrial AIS data base.

\section{FINANCIAL SUPPORT}

The research leading to these results has been carried out under the framework of the project 'R\&D for the maritime safety and security and corresponding real time services'. The project started in January 2013 and is led by the Program Coordination Defence and Security Research within the German Aerospace Center (DLR).

\section{REFERENCES}

Anderson, J., Aulin, T., and Sundberg, C. (1986). Digital Phase Modulation. Series: Applications of Communications Theory. Springer.

Bouny, N., LeMaitre, J. and Millerioux, J.-P. (2012). Results of measurement campaign for characterisation of AIS transmitters. 6th Advanced satellite multimedia systems conference (ASMS) and the 12th Signal processing for space communications workshop (SPSC), 258-265.

Brusch, S., Lehner, S., Fritz, T., Soccorsi, M., Soloviev, A. and van Schie, B. (2011). Ship surveillance with TerraSar-X. IEEE Transactions on Geoscience and Remote Sensing, 49, 10921103.

Burzigotti, P., Ginesi, A., and Colavolpe, G. (2010). Advanced receiver design for satellite-based AIS signal detection. 5th Advanced satellite multimedia systems conference (ASMA) and the 11th Signal processing for space communications workshop (SPSC), 1-8.

Carson-Jackson, J. (2012). Satellite AIS - Developing Technology or Existing Capability?. Journal of Navigation, 65, 303-321.

CEPT. (2013). Information Paper on VHF Data Exchange System (VDES), CPG PTC(13) INFO 16. Electronic Communications Committee, Tech. Rep.

Cervera, M. A., Ginesi, A. and Eckstein, K. (2011). Satellite-based vessel Automatic Identification System: A feasibility and performance analysis. Int. J. Satell. Commun. Network, 29, 117-142.

Changqing, L. and Xiaoqian, C. (2013). Inference of Single Vessel Behaviour with Incomplete Satellite-based AIS Data. Journal of Navigation, 66, 813-823.

Clazzer, F., Munari, A., Plass, S. and Suhr, B. (2014a) On the Impact of Coverage Range on AIS Message Reception at Flying Platforms. 7th Advanced satellite multimedia systems conference (ASMS) and the 13th Signal processing for space communications workshop (SPSC).

Clazzer, F., Munari, A., Berioli, M. and Lazaro Blasco, F. (2014b). On the Characterization of AIS Traffic at the Satellite. MTS/IEEE OCEANS - Taipei.

Eriksen, T., Høye, G., Narheim, B., and Meland, B. J. (2006). Maritime traffic monitoring using a space-based AIS receiver. Acta Astronautica, 58, 537-549.

EU PASTA MARE Project. (2010a). Technical Note 4.1: Vessel Density Mapping. Oct.

EU PASTA MARE Project. (2010b). Technical Note 15: Flight Trial Report, Issue 3. Oct.

exactEarth. (2013). exactEarth further Expands its Fleet of AIS Satellites. http://www.exactearth.com/news/2013-11-26/. Accessed 09 May 2014.

Filjar, R., Desic, S., Pokrajac, D. and Cubic, I. (2005) Internet AIS. Journal of Navigation, 58, 197206.

Greidanus, H., Alvarez, M., Eriksen, T., Argentieri, P., Cokacar, T., Pesaresi, A., Falchetti, S., Nappo, D., Mazzarella, F. and Alessandrini, A. (2013). Basin-wide maritime awareness from multi- 
source ship reporting data. International Journal on Marine Navigation and Safety of Sea Transportation, 7, 185-192.

Høye, G. K., Eriksen, T., Meland, B. J., and Narheim, B. T. (2008). Space-based AIS for global maritime traffic monitoring. Acta Astronautica, 62, 240-245.

IALA. (2003). Technical clarifications on ITU recommendation ITU-R M.1371-1, Edition 1.4.

IMO. (2002). International Convention for the Safety Of Life At Sea (SOLAS), Chapter V Safety of Navigation, Regulation 19.2.4.

ISO. (1993) ISO/IEC 3309:1993: Information technology - telecommunications and information exchange between systems - High-level data link control (HDLC) procedures - frame structure.

ITU-R M.1371-4. (04/2010). Technical characteristics for an automatic identification system using time-division multiple access in the VHF maritime mobile band. Radio Communications Study Group 8.

Mengali, U. and D’Andrea, A. (1997). Synchronization Techniques for Digital Receivers. Series: Applications of Communications Theory, Springer.

Morelli, M. and Mengali, U. (1998). Feedforward carrier frequency estimation with MSK-type signals. IEEE Communications Letters, 2, 235-237.

Ou, Z. and Zhu, J. (2008) AIS database powered by GIS technology for maritime safety and security. Journal of Navigation, 61, 655-665.

Pauluzzi, D. and Beaulieu, N. (2000). A comparison of SNR estimation techniques for the AWGN channel. IEEE Transactions on Communications, 48, 1681-1691.

Plass, S., Berioli, M., Hermenier, R., Liva, G. and Munari, A. (2013). Machine-to-machine communications via airliners. Transactions on Emerging Telecommunications Technologies, 24, $427-440$.

Plass, S. and Hermenier, R. (2014) Study on worldwide detection of AIS signals via airliners. MTS/IEEE OCEANS - Taipei.

Plass, S., Poehlmann, R., Dammann, A., Gentner, C. (2014) Investigations on AIS Signal Reception on Aircraft at Higher Altitudes. MTS/IEEE OCEANS - Taipei.

Poehlmann, R. (2014) Analysis of AIS data records. Bachelor Thesis, Technical University Munich, Germany.

Prevost, R., Coulon, M., Bonacci, D., LeMaitre J., Millerioux, J.-P., and Tourneret, J.-Y. (2012). Interference mitigation and error correction method for AIS signals received by satellite. Proceedings of the 20th European Signal Processing Conference (EUSIPCO), 46-50.

te Hennepe, F., Rinaldo, R., Ginesi, A., Tobehn, C., Wieser, M., Olsen, Ø., Helleren, Ø., Challamel, R. and Storesund, F. (2010). Space-based detection of AIS signals: Results of a feasibility study into an operational space-based AIS system. 5th Advanced satellite multimedia systems conference (ASMA) and the 11th Signal processing for space communications workshop (SPSC), $17-24$.

Wahl, T., Høye, G. K., Lyngvi, A. and Narheim, B. T. (2005) New possible roles of small satellites in maritime surveillance. Acta Astronautica, 56, 273-277.

Wahl, T. and Høye, G. K. (2003) New possible roles of small satellites in maritime surveillance. Proceedings of Fourth IAA Symposium on Small Satellites for Earth Observation, Berlin.

Yang, C., Hu, Q., Tu, X. and Geng, J. (2012). An integrated vessel tracking system by using AIS, Inmarsat and China Beidou navigation satellite system. International Journal on Marine Navigation and Safety of Sea Transportation, 6, 175-178.

Zhi, Z., Kefeng, J., Xiangwei, X., Huanxin, Z. and Shilin, Z. (2014). Ship Surveillance by Integration of Space-borne SAR and AIS - Review of Current Research. Journal of Navigation, 67, pp 177189. 